\title{
Groupement de recherche européen (GdRE) du CNRS « Terres cuites architecturales et nouvelles méthodes de datation », bilan 2010
}

Pierre Guibert et Christian Sapin

\author{
(2) OpenEdition \\ Journals \\ Édition électronique \\ URL : https://journals.openedition.org/cem/11946 \\ DOI : 10.4000/cem.11946 \\ ISSN : 1954-3093 \\ Éditeur \\ Centre d'études médiévales Saint-Germain d'Auxerre
}

Référence électronique

Pierre Guibert et Christian Sapin, « Groupement de recherche européen (GdRE) du CNRS «Terres cuites architecturales et nouvelles méthodes de datation », bilan 2010 », Bulletin du centre d'études médiévales d'Auxerre / BUCEMA [En ligne], 15 | 2011, mis en ligne le 27 mars 2013, consulté le 22 septembre 2022. URL : http://journals.openedition.org/cem/11946 ; DOI : https://doi.org/10.4000/ cem. 11946

Ce document a été généré automatiquement le 22 septembre 2022.

\section{(c)}

Creative Commons - Attribution - Pas d'Utilisation Commerciale - Partage dans les Mêmes Conditions 4.0 International - CC BY-NC-SA 4.0

https://creativecommons.org/licenses/by-nc-sa/4.0/ 


\title{
Groupement de recherche européen (GdRE) du CNRS « Terres cuites architecturales et nouvelles méthodes de datation », bilan 2010
}

\author{
Pierre Guibert et Christian Sapin
}

1 Il s'agit, en partant des terres cuites architecturales, et en particulier de la brique utilisée au sein de maçonneries mixtes avec de la pierre, d'avancer de nouveaux paramètres dans le champ des datations possibles, en posant les questions des remplois, des déplacements, des productions et, en définitive, de la place du composant dans une structure.

2 À partir d'édifices phares de l'Ouest de la France, nous avons montré comment le couplage des études de bâti et des méthodes de datation par thermoluminescence et archéomagnétisme sur les terres cuites aboutit à des informations particulièrement novatrices sur la chronologie de la production des briques, leur éventuel remploi ou sur leur production ad nove. Plus inattendu, ce couplage nous apporte des informations sur des modalités de leur fabrication.

3 Cette approche pluridisciplinaire peut, à terme, remettre en question ou au contraire confirmer les datations traditionnelles, reposant, souvent, uniquement sur un texte ou un élément stylistique. Pour cela, il importait de développer les méthodes et de les croiser. L'intérêt de ce programme est moins l'écart subsistant encore dans les datations, que la combinaison des méthodes utilisées. Il s'agit en l'occurrence d'associer carbone 14, thermoluminescence, luminescence optiquement stimulée, archéomagnétisme et, dans certains cas favorables, la dendrochronologie (tab. 1). 


\begin{tabular}{||l|l|l|l|l|}
\hline Méthode & $\begin{array}{l}\text { Événement } \\
\text { recherché }\end{array}$ & $\begin{array}{l}\text { Élément de } \\
\text { maçonnerie } \\
\text { concerné }\end{array}$ & Matériau support & Événement daté \\
\hline Carbone 14 & édification & mortier & $\begin{array}{l}\text { charbon de bois dans } \\
\text { le mortier }\end{array}$ & $\begin{array}{l}\text { fin de vie de l'élément } \\
\text { végétal }\end{array}$ \\
\hline $\begin{array}{l}\text { Thermoluminescence } \\
\text { (TL) ou Luminescence } \\
\text { optiquement stimulée } \\
\text { (OSL) }\end{array}$ & $\begin{array}{l}\text { production des } \\
\text { briques }\end{array}$ & brique & $\begin{array}{l}\text { quartz ou autres } \\
\text { minéraux prélevés au } \\
\text { cceur }\end{array}$ & dernier chauffage \\
\hline Archéomagnétisme & $\begin{array}{l}\text { production des } \\
\text { briques }\end{array}$ & brique & oxydes magnétiques & dernier chauffage \\
\hline $\begin{array}{l}\text { Luminescence } \\
\text { optiquement stimulée } \\
\text { (OSL) - datation de } \\
\text { surface }\end{array}$ & édification & brique & $\begin{array}{l}\text { quartz ou autres } \\
\text { minéraux en surface } \\
\text { de la brique }\end{array}$ & $\begin{array}{l}\text { dernière exposition à la } \\
\text { lumière }\end{array}$ \\
\hline \begin{tabular}{l} 
Dendrochronologie \\
\hline
\end{tabular} & édification & charpente & pièces de bois & abattage de l'arbre \\
\hline
\end{tabular}

Tab. 1 - Méthodes de datation mises en œuvre dans le cadre du GdRE. L'application de l'OSL à la datation de surface est en cours de développement.

4 La finalité pour les sciences humaines, en associant des spécialistes de chronologie et de caractérisation minérale, des historiens de l'art et des archéologues, est de proposer une autre idée de l'histoire du monument. Celui-ci ne peut être considéré comme figé dans son élaboration, car il révèle aussi les transformations sociales, religieuses et politiques de son temps. Il s'agit ainsi de mieux dater pour mieux comprendre.

\section{Événements marquants et travaux de datation en cours (2010-2011)}

5 Le GdRE TCA a été marqué en 2010 par plusieurs actions internationales. La réunion plénière de Catane (Italie) a permis de mettre en évidence la complexité de l'organisation d'expériences comparatives inter-laboratoires. Il s'agit souvent d'analyser les pratiques acquises sur le long terme dans chacune des équipes et qui peuvent aboutir à une divergence de résultats. On montre surtout que la datation par luminescence ne peut être envisagée comme une méthode de routine, tant que l'ensemble des facteurs dispersifs n'aura pas été pris en compte et qu'une méthodologie concertée n'aura pas été définie pour restreindre les erreurs.

6 Des présentations des travaux du GdRE ont été effectuées dans des colloques internationaux : ISA 2010 à Tampa, UK luminescence dating, colloque d'archéologie du bâti à Liège en novembre 2010. Sur le plan de la recherche méthodologique, d'importantes avancées ont été réalisées sur la question des hyper-vieillissements de certaines datations opérées par la méthode des inclusions de quartz. Les études archéomagnétiques sur l'archéo-intensité à des fins d'étalonnage du champ magnétique terrestre sont en cours sur l'ensemble des sites. Nous rappelons que des échantillons communs pour la datation par luminescence et par magnétisme ont été prélevés.

7 Le programme sur les sites comprend l'achèvement des datations par luminescence Aquitaine, Chartres, Saint-Martin d'Angers (édifices anciens), Mayenne - et la réalisation des datations des TCA dans la région Rhône-Alpes: La Manécanterie de Lyon, Saint-Romain-en-Gal près de Vienne et les vestiges de l'abbaye de Savigny. Les résultats sur Saint-Irénée de Lyon devraient faire l'objet d'une publication prochaine (tab. 2). 


\begin{tabular}{|c|c|c|}
\hline Saint-Irénée, Lyon & \multirow{5}{*}{$\begin{array}{l}\text { Doctorat Armel } \\
\text { Bouvier } \\
\text { (alloc. ministère) }\end{array}$} & $\begin{array}{l}\text { Publication à soumettre à } \\
\text { Archéosciences }\end{array}$ \\
\hline Cathédrale de Chartres & & $\begin{array}{l}\text { Datations aujourd'hui } \\
\text { abouties, des mesures } \\
\text { complémentaires de } \\
\text { radioactivité des quartz } \\
\text { utilisés pour la mesure de dose } \\
\text { ont été réalisées en janvier } \\
2011 \text { par ICP-MS }\end{array}$ \\
\hline Saint-Seurin, Bordeaux & & $\begin{array}{l}\text { Datations TL/OSL et } \\
\text { radiocarbone abouties, } \\
\text { interprétations et publication } \\
\text { en cours }\end{array}$ \\
\hline $\begin{array}{l}\text { Notre-Dame-de-la-Place, } \\
\text { Bordeaux }\end{array}$ & & $\begin{array}{l}\text { Datations TL/OSL abouties, } \\
\text { échantillons partagés avec } \\
\text { Milan et Catane ; Durham n'a } \\
\text { pas observé de signal OSL } \\
\text { exploitable }\end{array}$ \\
\hline $\begin{array}{l}\text { Sainte-Quitterie, Aire-sur- } \\
\text { l'Adour }\end{array}$ & & $\begin{array}{l}\text { Datations TL/OSL abouties, } \\
\text { interprétation en cours }\end{array}$ \\
\hline Château de Mayenne (53) & \multirow{4}{*}{$\begin{array}{l}\text { Post-doctorat } \\
\text { Sophie Blain } \\
\text { (alloc. PRES } \\
\text { Bordeaux / } \\
\text { Conseil régional } \\
\text { d'Aquitaine) }\end{array}$} & Datations TL/OSL en cours \\
\hline $\begin{array}{l}\text { Monastère de Castelletto- } \\
\text { Cervo (BI) }\end{array}$ & & $\begin{array}{l}\text { Datations TL/OSL abouties, } \\
\text { interprétation en cours }\end{array}$ \\
\hline Prieuré de Savigny (69) & & Datations TL/OSL abouties \\
\hline $\begin{array}{l}\text { Lyon, La Manécanterie } \\
\text { Saint-Romain à Saint- } \\
\text { Romain-en-Gal }\end{array}$ & & Datations TL/OSL en cours \\
\hline
\end{tabular}

Tab. 2 - Liste des sites en cours d'étude chronologique (état en avril 2011), programmes de travail financés spécifiquement et état d'avancement. L'étude archéomagnétique est en cours sur l'ensemble des sites. Nous rappelons que des échantillons communs pour la datation par luminescence et par magnétisme ont été prélevés systématiquement.

8 Par ailleurs, doivent se finaliser les travaux d'intercomparaison sur le prieuré de Castelletto-Cervo (Italie) et la publication commune sur ce site clunisien entre les équipes internationales.

\section{Publications}

- S. blain, P. guibert, I. Bailiff, A. Bouvier et M. BAylé, "An intercomparison study of luminescence dating protocols and techniques applied to medieval brick samples from Normandy, France », Quaternary Geochronology, 5 (2010), p. 311-316.

- A. M. Gueli, G. Stella, S. O. Troja, G. Burrafato, D. Fontana, G. M. Ristuccia et

A. R. ZuCCARELLO, « Historical buildings : luminescence dating of fine grains from bricks and mortar », Il Nuovo Cimento, 125B (2010, série 5-6), p. 719-729.

- S. blain, P. guibert, D. Prigent, P. Lanos, C. Oberlin, C. Sapin, A. Bouvier et P. Dufresne, «Dating methods combined to building archaeology: the contribution of thermoluminescence to the case of the bell tower of St Martin's church, Angers (France) ", Geochronometria, à paraître.

- A. Bouvier, G. PINTO, P. GUIBERT, D. NicolAS-MÉRY et M. BAYLÉ, « Luminescence dating applied to medieval architecture: the north east tower of Avranches'dungeon (Manche, France)", Archéosciences, à paraître.

-S. BLAIN, L'utilisation des terres cuites architecturales dans des édifices religieux du haut Moyen Âge de l'Ouest de la France et du Sud de l'Angleterre : une approche par la datation par luminescence / The use of Ceramic Building Materials in early medieval religious buildings: Approach by Luminescence dating, publication de la thèse dans British Archaeological Records, 2011. 